\title{
Quartz-Crystal-Microbalance Study for Dissolution of Resist Polymers
}

\author{
Minoru Toriumi \\ Laboratory for Interdisciplinary Science and Technology, Tokyo, Japan \\ minoru@lartist.jp
}

\begin{abstract}
The quartz crystal microbalance (QCM) method was used to obtain precise information about the dissolution behavior of resist polymers. The time evolutions of the resonance frequency and the impedance during the dissolution were measured using the QCM method. The data were simulated using the characteristic matrix method on a four-layer model. The matrix method showed Sauerbrey's relation to be invalid during the formation and dissolution of the swelling or gel layer, although Sauerbrey's relation was valid during the other development time with the constant thickness of the swelling layer. There are two kinds of swelling behaviors. In the case of thinner thicknesses of swelling layer, the rigidity of swelling layer is constant. The larger thickness of swelling layer shows the large decrease of rigidity during the development. The residue after the development shows also larger change of rigidity of a swelling layer. The characteristic-matrix analysis has shown the validity of quantitative analysis in QCM experiments.
\end{abstract}

Keyword s : development, swelling, QCM, characteristic matrix method

\section{Introduction}

Resist is one of key materials to determine the resolution, thought-put, process for the microlithography. There are many requirements such as sensitivity, dry-etching durability, and solubility in a developer. One of the most important characteristics of resist materials is dissolution behavior during a development. A resist relief image can be formed by developing a latent image using an exposure system. Developing resist materials is critical to determine the resolution, line edge roughness (LER), collapse of resist patterns, and so on.

In order to study the dissolution behavior, optical interferometers have been conventionally used in the microlithography. However, the optical interference measurement can not be used to study the dissolution behavior of resist films when film thickness becomes thinner. Especially studying the swelling behavior during the development of resist materials is very difficult, because the signal intensity becomes weaker and more complicated due to the optically indistinct swelling-interface.

The quartz crystal microbalance (QCM) method is used in vacuum science and technology for measuring mass increase caused by deposition or mass loss caused by irradiated energetic particles. The QCM method has been applied to resist studies of the diffusion, sorption, and desorption of small molecules to and from resist films [1], dissolution of arylate polymers [2], dissolution in supercritical carbon dioxide [3], sissolution mechanis [4], and water absorption for immersion lithography [5-8],

The QCM method has been used to measure the dissolution kinetics of thin films in liquid media [9]. The QCM method works by dissolving the resist film on a quartz crystal oscillator. The oscillator vibrates at a fundamental resonance frequency. As the mass dissolves away into the developer from the surface of the quartz oscillator, the resonance frequency of the oscillator increases. Sauerbrey's equation, which means the linear relationship between mass and frequency, has been used for the resist films. In recent studies using QCM dissolution rate monitors, several polymer systems have been studied which allow permeation of the liquid developer components into the film leading to large swelling prior to dissolution [10-12].

The physical model of a dissolving film on a quartz oscillator was proposed to analyze QCM data [13]. Figure 1 shows the four-layer model 
which consists of a quartz substrate, a dry polymer film, an interfacial layer or a swelling gel-layer, and a liquid. The model relates the changes in the structure and viscoelastic properties of the

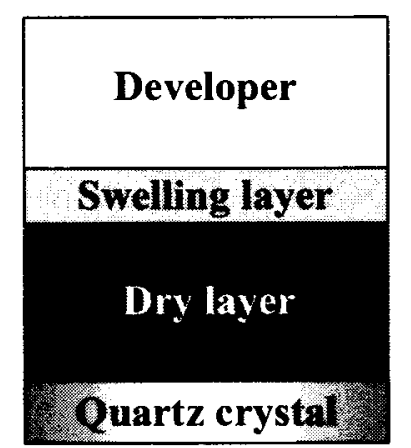

Fig. 1. Four-layer model to elucidate polymer dissolution in developer.

dissolveing acidic-polymer film as it dissolves or swells in an aqueous alkaline solution. If the model exhibits swelling, the interfacial transition layer exhibits a greatly reduced elastic modulus and lowered viscosity compared with the solid polymer before swelling. The model was mechanically treated using non-linear simultaneous equations, and it elucidated the swelling behavior of a norbornene polymer [14]. Taxonomy of the dissolution processes has been proposed for representative resist copolymers, measured using QCM frequency-resistance analysis and optical reflectance [15]. The dissolution behavior is supposed to be determined only by two variables, the base diffusion and the relative deprotonation rate constant of the polymer.

The four-layer model was also analyzed using the characteristic matrix method based on an equivalent electric circuit [16]. It enables to convert the simultaneous equation problem into the equivalent simple matrix operation. This characteristic matrix method derives a simple analytical equation. We used this analytical equation to simulate the QCM data and to obtain quantitative information on the development kinetics of resist materials and to show the validity of Sauerbrey's relation and the effects of the swelling layer on the QCM data.

\section{Method}

\subsection{Theory [16]}

In the QCM method, the impedance of a sample system is measured during the development process simultaneously with the shift of resonance frequency. In the characteristic matrix analysis, the sample impedance, $Z$, loaded on the crystal is described by

$$
\begin{aligned}
Z= & j \omega h_{s} \rho_{s}+ \\
& Z^{(2)} \frac{Z^{(3)} \cosh \left(\gamma^{(2)} h_{f}\right)+Z^{(2)} \sinh \left(\gamma^{(2)} h_{f}\right)}{Z^{(2)} \cosh \left(\gamma^{(2)} h_{f}\right)+Z^{(3)} \sinh \left(\gamma^{(2)} h_{f}\right)}
\end{aligned}
$$

where $Z^{(2)}, Z^{(3)}$, and $\gamma^{(2)}$ are defined as

$$
\begin{aligned}
Z^{(2)} & =\sqrt{\rho_{f} G_{f}} \\
Z^{(3)} & =\sqrt{\frac{\omega \rho_{l} \eta_{l}}{2}}(j+1) \\
\gamma^{(2)} & =j \omega \sqrt{\frac{\rho_{f}}{G_{f}}}
\end{aligned}
$$

where, $j$ is the imaginary unit, $\omega$ is the angular frequency, $h, \rho, G$, and $\eta$ are a thickness, a density, a shear modulus, and a viscosity, respectively, and the subscripts $s, f$, and $l$ represent a quartz substrate, a dry film, and a liquid medium, respectively.

The impedance spectrum is simulated using Eq. (1). The resonance frequency is determined as the frequency to minimize the impedance. The impedance to be discussed here is the value at the resonance frequency.

\subsection{Apparatus for Quartz Crystal Micro-} balance

The instrument is shown schematically in Fig. 2. In this study the QCM measurements were performed on the RQCM, Maxtek, Inc. The polymer samples were spun cast onto a one-inch AT-cut piezoelectric quartz crystal plate with a nominal characteristic frequency of $5 \mathrm{MHz}$ and was post-application baked. After coating, the sample-coated crystal was mounted in the home-made liquid flow cell. The sensor was oscillated with a nominal 
characteristic frequency by the RQCM. The developer is pumped through the flow cell from the computer-controlled syringe injector. The frequency and impedance changes of the samples were measured during the development of the alkaline aqueous solution using the RQCM with a time resolution of $20 \mathrm{~Hz}$.

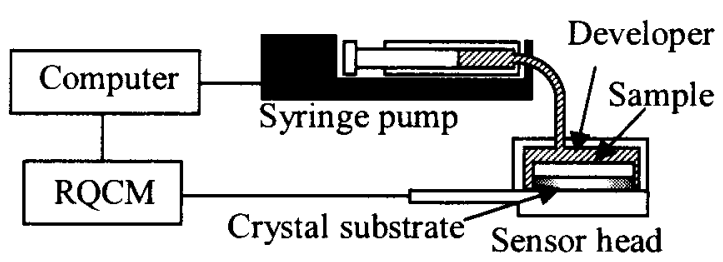

Fig. 2. QCM apparatus to measure the frequency and impedance of sample.

\section{Results and Discussion}

\subsection{Sauerbrey's relation}

Simple model for dissolution of polymer films is a model without forming a swelling layer during development. This non-swelling dissolution is one of the important properties which may reduce the line-edge roughness of resist patterns. The characteristic matrix method was applied to evaluate the model shown in Fig. 3. The solid line shows the thickness change of dry layer during the

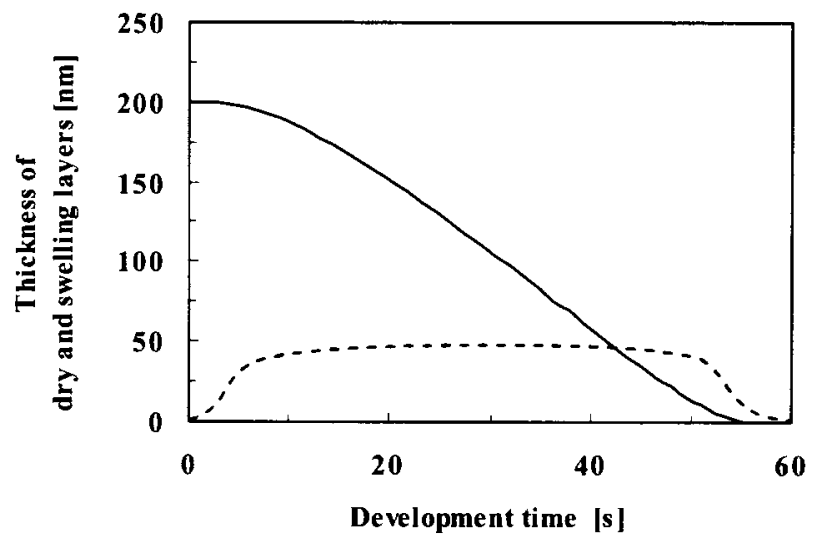

Fig. 3. Assumed changes of a dry layer (solid curve) and a swelling layer (dashed curve) of the four-layer model during development. development. There is no swelling layer during the development in this model. The resonance frequency was calculated and the frequency was correlated well with the change of the film thickness, having a correlation factor of 0.9998 . The shift of the resonance frequency was proportional to the thickness changes during the dissolution. This linear relation is Sauerbrey's relation that the resonance frequency of a quartz crystal is directly dependent on the mass of the crystal, including the mass of any film coated onto the crystal's surface. The conventional QCM analysis works on this simple Sauerbrey's relation.

\subsection{Effects of a swelling layer}

The dashed curve in Fig. 3 shows the thickness change of a swelling layer. The film was assumed to form a swelling layer when first contacting the developer, to dissolve in the developer with the swelling layer, and to disappear after the dissolution of the swelling layer, as shown in Fig. 3. The swelling layer was assumed to maintain a constant thickness corresponding to the case II diffusion of a resist film [13]. The swelling thickness was set to $50 \mathrm{~nm}$ to make the swelling behavior clear. The characteristic matrix method was applied to evaluate the effects of a swelling

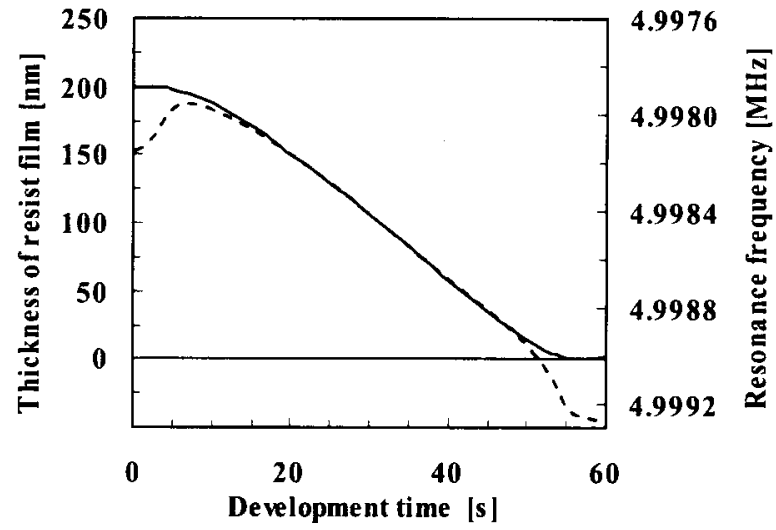

Fig. 4. Changes of resonance frequency (a dotted line) calculated by the characteristic matrix method using thickness change of summation of dry and swelling layers (a dashed line) during development. 
layer using dry and swelling layers in Fig. 3. The calculated results are shown in Fig. 4. The frequency was well proportional to the film thickness in the bulk region where a swelling layer keeps a constant thickness. In the plateau region Sauerbrey's relation still comes into effect. However, Suerbrey's rule does not hold true in the case of thickness change of a swelling layer such as increase or decrease of swelling layer. It should be noted that the film thickness is not proportional to the shift of resonance frequency in these regions.

Figure 5 shows a QCM trace of an acrylate polymer film. Resonance frequency and impedance of the film were measured during development. The characteristic matrix method was applied to the data. The monotonic decrease of resonance frequency indicates the monotonic swelling during the development. The impedance shows constant value during the development. The calculated swelling behavior was shown in Fig. 6. The film thickness during the development is roughly proportional to the shift of resonance frequency under the condition that the thickness change of a sample film is small during the development.

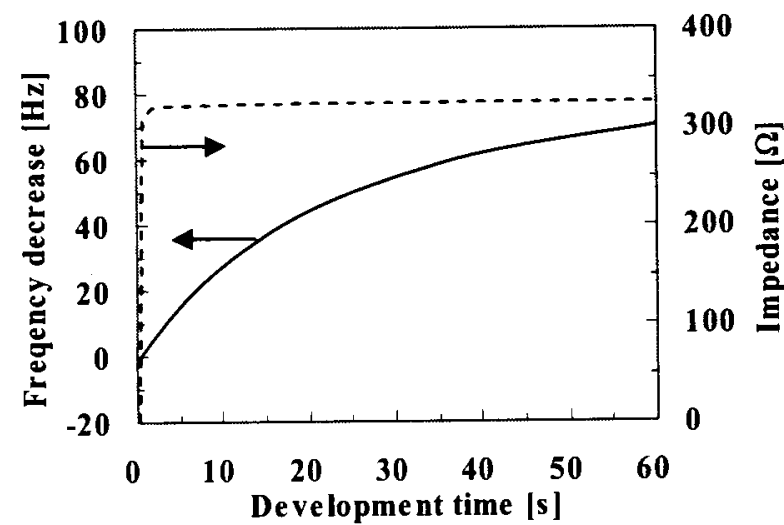

Fig. 5. QCM traces of resonance frequency (solid curve) and impedance (dashed curve) of an acrylate film which shows a constant impedance.

\subsection{Effects of rigidity in a swelling layer}

Figure 5 shows a QCM trace of an acrylate polymer film which shows a monotonic increase of impedance curve.

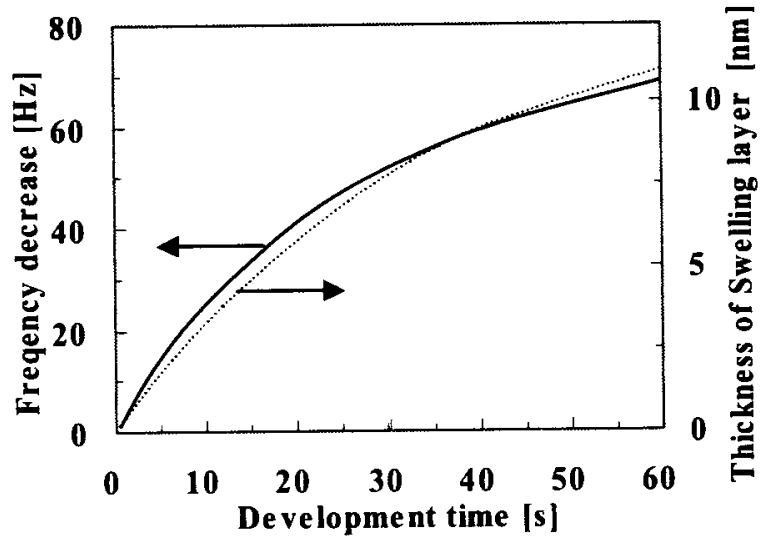

Fig. 6. Simulated resonance frequency (solid curve) and thickness of a swelling layer (dotted curve) simulated by the matrix method using QCM data of Fig. 5.

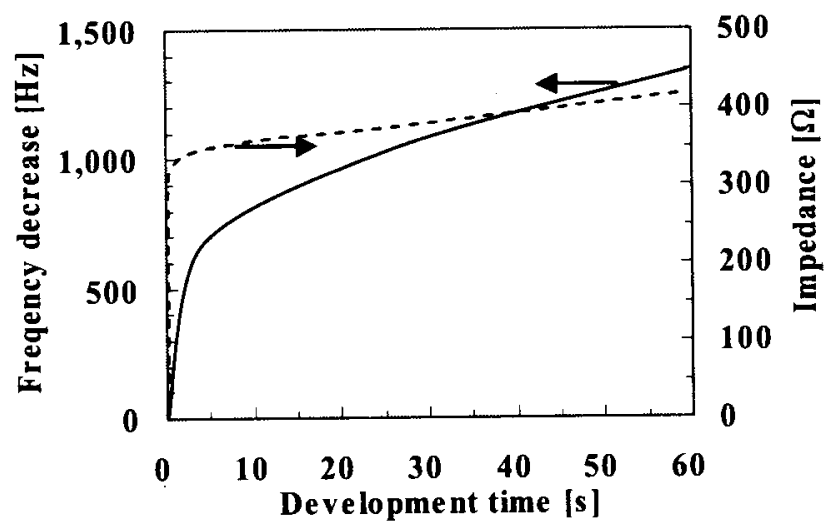

Fig. 7. QCM traces of resonance frequency (solid curve) and impedance (dashed curve) of an acrylate film which shows a monotonic increase in impedance.

The data shows large change of resonance frequency at the onset followed by monotonic change. The property, rigidity, of a swelling layer should be considered in this case. The results simulated by the matrix method are shown in Fig. 8 . The rigidity of a swelling layer decreases at the initial formation stage of the swelling layer. The rigidity decrease is reasonable because the formation of a gel layer by absorbing the developer. 
Apparently the film thickness during the development is not proportional to the shift of resonance frequency under the condition that the rigidity of resist film changes during development.

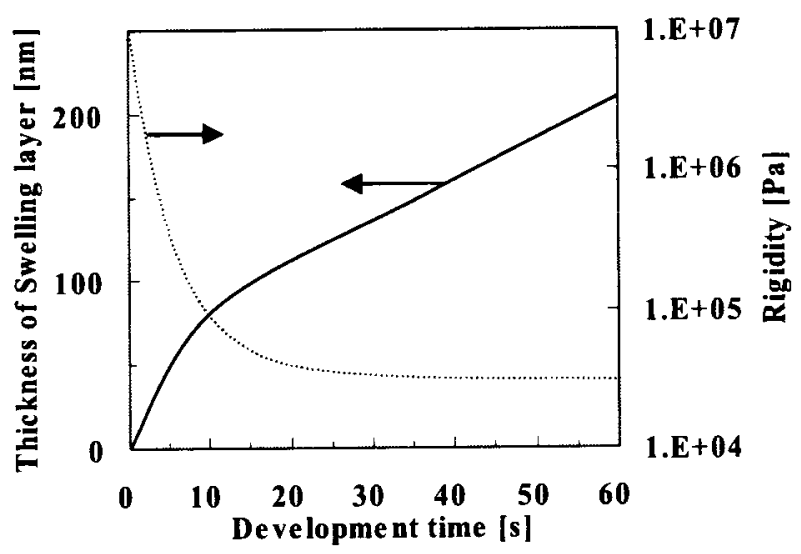

Fig. 8. Change of thickness of swelling layer (dotted curve) and rigidity (dotted curve) simulated by the matrix method using the QCM data in Fig. 7.

\subsection{Effects of Residue during development}

Figure 9 shows a QCM trace of a polymer film which shows a large change in resonance frequency. It indicates residues after development. The results simulated by the matrix method are shown in Fig. 10.

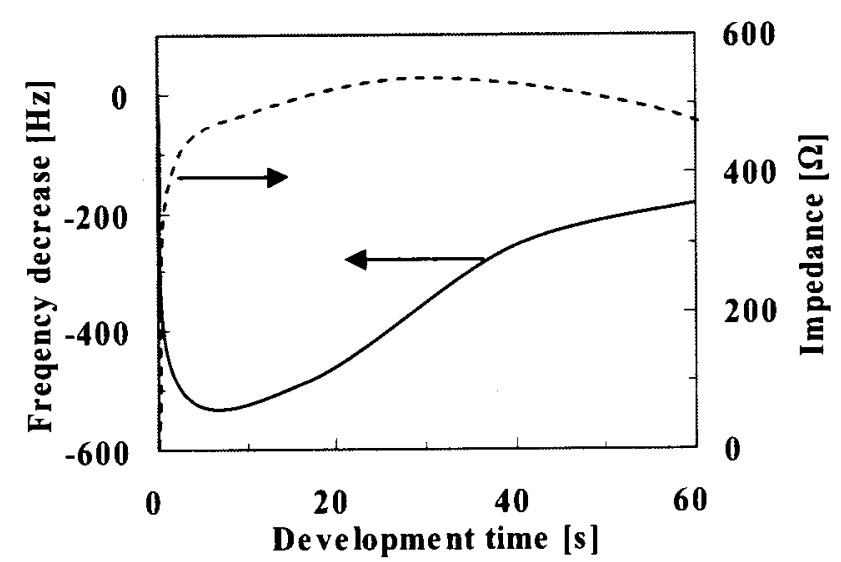

Fig. 9. QCM traces of resonance frequency (solid curve) and impedance (dashed curve) of a polymer film which shows residues after development.

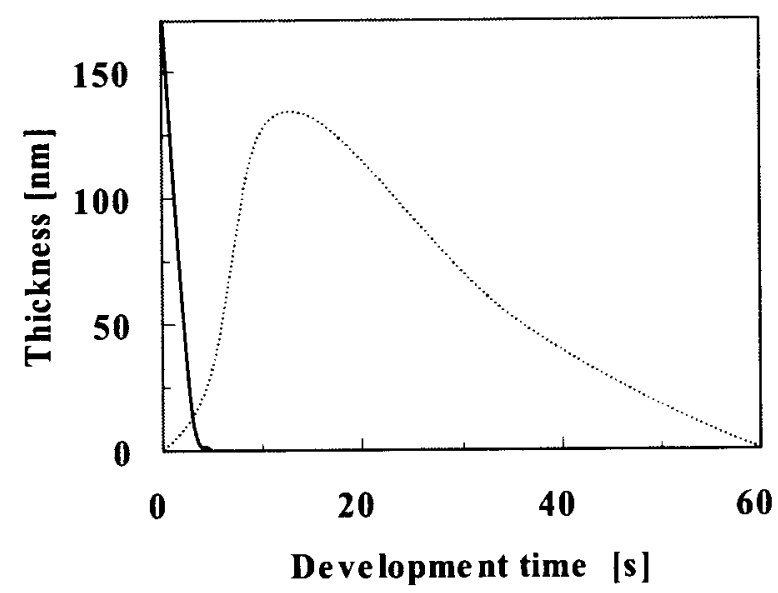

Fig. 10. Change of thickness of dry layer (a solid curve) and swelling layer (a dotted curve) simulated by the matrix method using the QCM data in Fig. 9.

The film dissolved away rapidly just in the beginning of the development and large swelling layer grew on during the dissolution. The rigidity of the swelling layer was also decreased from $10^{7}$ to $10^{4} \mathrm{~Pa}$ within 5 seconds, which synchronize the dissolution of the dry layer. Although the dry layer dissolves rapidly in the developer, the residues show slower dissolution after the swelling. The polymer may segregate the soluble components and non-soluble components during the early stage of the development. Soluble components in the polymer film dissolve preferably in the developer and the insoluble components accumulates and form the residues which show the slow dissolution.

\section{Conclusion}

The quartz crystal microbalance (QCM) method is valid for studying the dissolution behavior of polymer films coated on a crystal oscillator. The simultaneous measurement of resonance frequency and impedance gives precise information on the dissolution phenomena. QCM data were simulated using the characteristic matrix method on a fourlayer model. The matrix method showed Sauerbrey's relation to be invalid during the formation and dissolution of the swelling or gel layer, although Sauerbrey's relation was valid during the other development time with the constant thickness of the swelling layer. 
There are two kinds of swelling behaviors. In the case of thinner thicknesses of swelling layer, the rigidity of swelling layer is constant. The larger thickness of swelling layer shows the large decrease of rigidity during the development. The residue after the development shows also larger change of rigidity of a swelling layer.

The characteristic-matrix analysis has shown the validity of quantitative analysis in QCM experiments.

\section{References}

1. L. Singh, P. J. Ludovice, and C. L. Henderson, Advances in Resist Technology and Processing XXI, J. L. Sturtevant, Editor, SPIE Vol. 5376, (2004) 369.

2. M. Kitayama, I. Aratani and M. Toriumi, Advances in Resist Technology and Processing XXIII, Qinghuang Lin, Editor, SPIE Vol. 6153, (2006) 61532A.

3. A. E. Zweber, and R. G. Carbonell, Advances in Resist Technology and Processing XXIII, Qinghuang Lin, Editor, SPIE Vol. 6153, (2006) 61534C-1.

4. A. Rao, S. Kang, B. D. Vogt, V. M. Prabhu, E. K. Lin, W.-L. Wu, K. Turnquest and W. D. Hinsberg, Advances in Resist Technology and Processing XXIII, Qinghuang Lin, Editor, SPIE Vol. 6153, (2006) 615310-1.

5. N. Stepanenko, H.-W. Kim, S. Kishimura, D. V. D. Heuvel, N. Vandenbroeck, M. Kocsis, P. Foubert, M. Maenhoudt, M. Ercken, F. V. Roey, R. Gronheid, I. Pollentier, D. Vangoidsenhoven, C. Delvaux, C. Baerts, S. O'Brien, W. Fyen, G. Wellsa, Advances in Resist Technology and Processing XXIII, Qinghuang Lin, Editor, SPIE Vol. 6153, (2006) 615304-1.

6. M. Toriumi, C. Matsubara, A. Otoguro, T.
Itani, Advances in Resist Technology and Processing XXIII, Qinghuang Lin, Editor, SPIE Vol. 6153, (2006) 615311-1.

7. A. Otoguro, J.-W. Lee, T. Itani, K. Fujii, T. Funakoshi, T. Sakai, K. Watanabe, M. Arakawa, H. Nakano and M. Kobayashi, Advances in Resist Technology and Processing XXIII, Qinghuang Lin, Editor, SPIE Vol. 6153, (2006) 61531 P-1.

8. N. Shirota, Y. Takebe, T. Sasaki, and O. Yokokoji, M. Toriumi, and H. Masuhara, Advances in Resist Technology and Processing XXIII, Qinghuang Lin, Editor, SPIE Vol. 6153, (2006) 615324-1.

9. W. D. Hinsberg, C. G. Willson, and K. K. Kanazawa, J. Electrochem. Soc., 133, (1986) 1448.

10. M. Toriumi, T. Ohfuji, M. Endo, and H. Morimoto, J. Photopolymer. Sci. Technol. Japan, 12, (1999) 545.

11. H. Ito, R. Allen, J. Opitz, T. Wallow, H. Truong, D. Hofer, P. Varanasi, G. Jordhamo, S. Jayaraman, and R. Vicari, Proc. Photo-Optical Instr. Eng., 3999, (2000) 2.

12. T. Wallow, W. Chan, W. Hinsberg, and S-W. Lee, Advances in Resist Technology and Processing $X I X, \mathrm{~T}$. H. Fedynyshyn, Editor, SPIE Vol. 4690, (2002) 299.

13. W. Hinsberg, S-W. Lee, H. Ito, D. Horne, and K. Kanazawa, Advances in Resist Technology and Processing XVIII, F. M. Houlihan, Editor, SPIE Vol. 4345, (2001) 1.

14. S-W. Lee, W. Hinsberg, and $\mathrm{K}$. Kanazawa, Anal. Chem., 74, (2002) 125.

15. W. Hinsberg, F. Houle, and H. Ito, Advances in Resist Technology and Processing XXI, SPIE Vol. 5376, (2004) 352.

16. M. Toriumi, T. Itani, J. Yamashita, T. Sekine, and K. Nakatani, Advances in Resist Technology and Processing XIX, T. $\mathrm{H}$. Fedynyshyn, Editor, SPIE Vol. 4690, (2002) 904. 